\title{
A multiprogram approach to real-time experimentation*
}

\author{
H. J. DURRETT, JR. $\dagger$ \\ The Computer Laboratory for Instruction in Psychological Research \\ University of Colorado, Boulder, Colorado 80302
}

\begin{abstract}
A technique for implementing real-time experiments utilizing several separate and independent programs is discussed. The technique can be employed when the experimental procedure is divided into real-time and nonreal-time program units. These program units are executed in the most appropriate computing environment, and the resulting output is directed to a sequential file. The nonreal-time program issues commands to the real-time program by means of keywords that are inserted in the sequential file output at those points where real-time operations are required. These commands are interpreted by the real-time program which processes the sequential file. While the nonreal-time program is experiment-specific, the real-time program is independent of the particular experiment performed. The applications of this multiprogram approach to real-time experimentation are considered.
\end{abstract}

The Computer Laboratory for Instruction in Psychological Research must simultaneously support several real-time experiments. The laboratory must do this with limited resources. The most precious of these resources is central processor memory, while the least precious is sequential file storage. Thus, when designing and implementing a new real-time application program, the minimization of memory requirements is of major importance and the use of sequential file storage is of minor importance.

A simple and efficient programming technique has been developed to achieve a maximum utilization of real-time resources. This technique employs several independent programs to implement a single experiment. These programs are serially executed at different times and in the most appropriate computing environments. All output from these programs is directed to sequential file storage, such as disk or tape. One of the programs issues commands to the other by means of keywords which are inserted in the sequential file output. Thus, the simple keyword-command system is used to "explain" the output to the program processing it. Since this technique is adaptable to any computing facility that possesses some type of sequential file storage device, and since it seeks to solve the common problem of limited real-time resources, it is of possible interest.

The successful implementation of a real-time experiment is possible only if the programs designed to execute the experiment efficiently utilize the resources available. This can be accomplished by employing a multiprogram approach in implementing the real-time experiment. The operations necessary to conduct an experiment are of two types: (1) those operations which do not require a real-time environment, and (2) those operations which do require a real-time environment.

\footnotetext{
*This research was conducted while the author was supported by National Science Foundation Grants GJ 453 and GB 34077 X.

tRequests for reprints should be sent to $H$. J. Durrett, Jr. Department of Psychology, University of Colorado, Boulder, Colorado 80302 .
}

The greater part of most programs designed to implement real-time experiments does not require a real-time environment. This part is present only to execute experiment-specific tasks, such as specialized input and output, material generation, and data acquisition. This nonreal-time part of an experimental program requires much of the available real-time resources. Since the nonreal-time part of an experimental program processes information much like a typical batch program, a discussion of its basic operations will be unnecessary. However, a detailed analysis of the basic operations performed by the real-time part of an experimental program will aid in the identification of what should or should not be executed in real time.

\section{BASIC REAL-TIME OPERATIONS}

The real-time part of most experimental programs can be characterized by 11 basic operations, which are repeatedly performed and which are independent of the particular experimental paradigm being executed. These basic operations can be organized into four sets of operations. For the purposes of this presentation, these operations will be given keyword names, which will serve to identify their functions. The keywords will have the form "!" followed by some word which is indicative of the operations represented.

\section{Acquisition of Information}

The first basic real-time operation is the acquisition of information used to control the real-time experimental processes. This information typically consists of timing parameters, solution criteria, and various limits placed on the number of trials and the duration of an experiment. This operation can be represented by a single keyword, !VALUE. The !VALUE keyword represents a command to acquire all information that is actively used in the real-time environment. This 


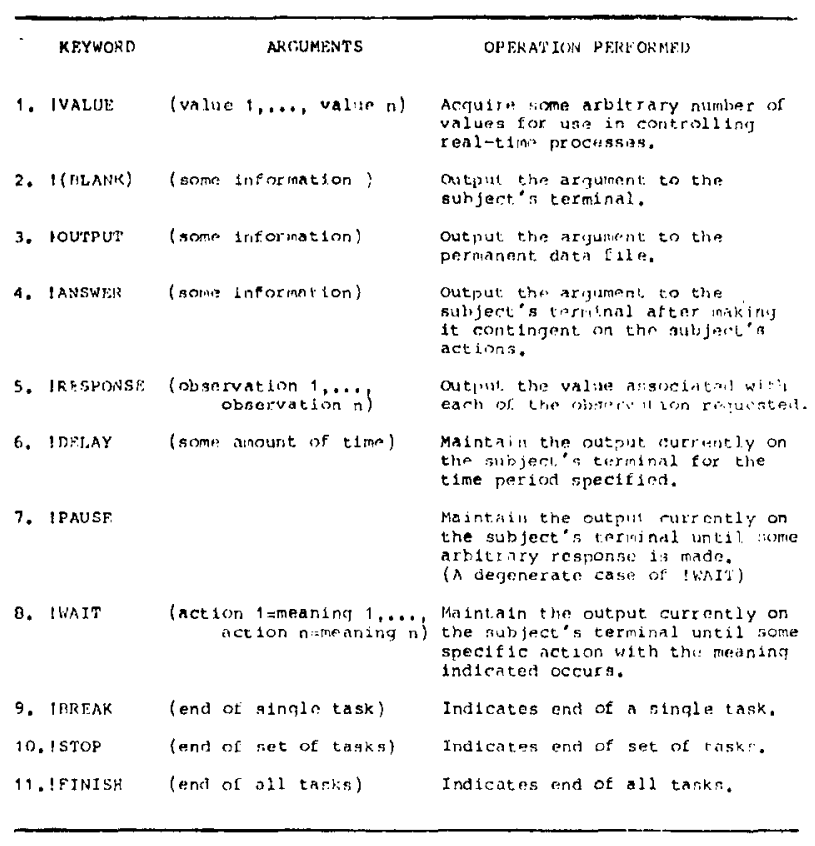

Fig. 1. Basic real-time operations and associated keywords.

command has some arbitrary number of arguments and can be written as

!VALUE (Value 1, Value 2, $\cdots$, Value n).

\section{Output of Information}

The second basic set of real-time operations concerns the output of information. This output is typically directed either to a permanent data file or to a S's terminal. Further, this output is either contingent or noncontingent on a S's actions. Thus, there are four output operations, which are represented by the keywords !(BLANK), !OUTPUT, !ANSWER, and !RESPONSE.

The !(BLANK) keyword represents a command to output a message to the S's terminal which is predetermined and which is not contingent on any action by the $\mathrm{S}$. This keyword can also be thought of as the "default mode" of the real-time program. That is, if no other operation is specified, output is directed to the S's terminal.

The !OUTPUT keyword represents a command to direct information to the permanent data file. This output is not contingent on a S's actions and is predetermined.

The !ANSWER keyword represents a command to direct output to the S's terminal which is contingent on the S's actions. The !ANSWER command requires one argument which is to be the reply to S's actions. This argument may be modified prior to being directed to the S's terminal, so that it reflects the S's actions. This command can be written as

!ANSWER (response to S's action).
Finally, the !RESPONSE keyword represents a command to direct output, which reflects a S's actions, to the permanent data file. This command has an arbitrary number of arguments which specify what observations about a S's actions are to be output. This command can be written

$$
\text { !RESPONSE (Observation 1, } \cdots \text {, Observation n). }
$$

\section{Timing of Output}

The third basic set of real-time operations involves the timing of output directed to a S's terminal. This timing can be done in three ways. It can be done such that the output is maintained for a predetermined amount of time, or until a $S$ makes an arbitrary response, or until a $S$ makes a specific response. These operations are represented by the keywords !DELAY, !PAUSE, and !WAIT.

The !DELAY keyword represents the timing operation used to maintain output for a predetermined amount of time. The keyword !DELAY requires a single argument specifying the amount of time to maintain the output. This command can be written

!DELAY (time period to maintain output).

The !PAUSE keyword represents the timing operation used to maintain output until the $\mathrm{S}$ makes some arbitrary response. It requires no arguments and is simply a degenerate form of the !WAIT operation.

The !WAIT keyword represents the timing operation used to maintain output until the $S$ acts in a specific way that has some relation to the output. The !WAIT keyword requires some arbitrary number of argument pairs which specify the acceptable actions and their meanings. This command can be written:

!WAIT (Action 1=Meaning 1, $\cdots$, Action n=Meaning n).

\section{Termination of Operations}

The fourth basic set of real-time operations involves the control of program execution. Real-time programs can control the presentation of a single task or sets of tasks. Thus, programs must identify the end of a single task, or the end of a set of tasks, or the end of all tasks. These termination operations can be represented by the commands: !BREAK, !STOP, and !FINISH.

The !BREAK command represents the end of a single task, the !STOP command represents the end of a set of tasks, and !FINISH represents the end of all tasks.

A summary of all of these 11 basic operations and their associated keyword commands is contained in Fig. 1.

\section{IMPLEMENTING A REAL-TIME EXPERIMENTAL PROGRAM}

Once the separate real-time and nonreal-time parts of an experimental program have been isolated, optimization of each can be considered. To clarify this, a 
representative programming example will be discussed. Consider a program designed to conduct concept identification experiments. These experiments employ lists of words as stimuli which a $\mathrm{S}$ is required to classify according to some relationship. The $S$ is informed of the correctness of the classification, and the procedure is repeated until some criterion number of correct trials is reached or the $S$ exceeds the number of trials or amount of time allowed to solve.

If such an experiment were implemented as a single self-contained program, its size and demand on real-time resources would make its use impractical. Thus, the nonreal-time part of the experiment must be isolated from the real-time part of the experiment.

The nonreal-time part often consists of the following: (1) the acquisition of experimental parameters, stimuli, and instructions, (2) the construction of the stimuli according to predetermined criteria, and (3) the identification of stimuli and their rules of classification. These operations can be performed by a single batch program, which would be executed in a nonreal-time environment. This program would generate all material to be presented to a $S$, assuming no solution was obtained. This nonreal-time program will be called the "generation" program. All of this output would be directed to a sequential file to be interpreted and processed by the real-time part of the experiment. However, wherever a real-time operation is required, a keyword command would be inserted. These keywords

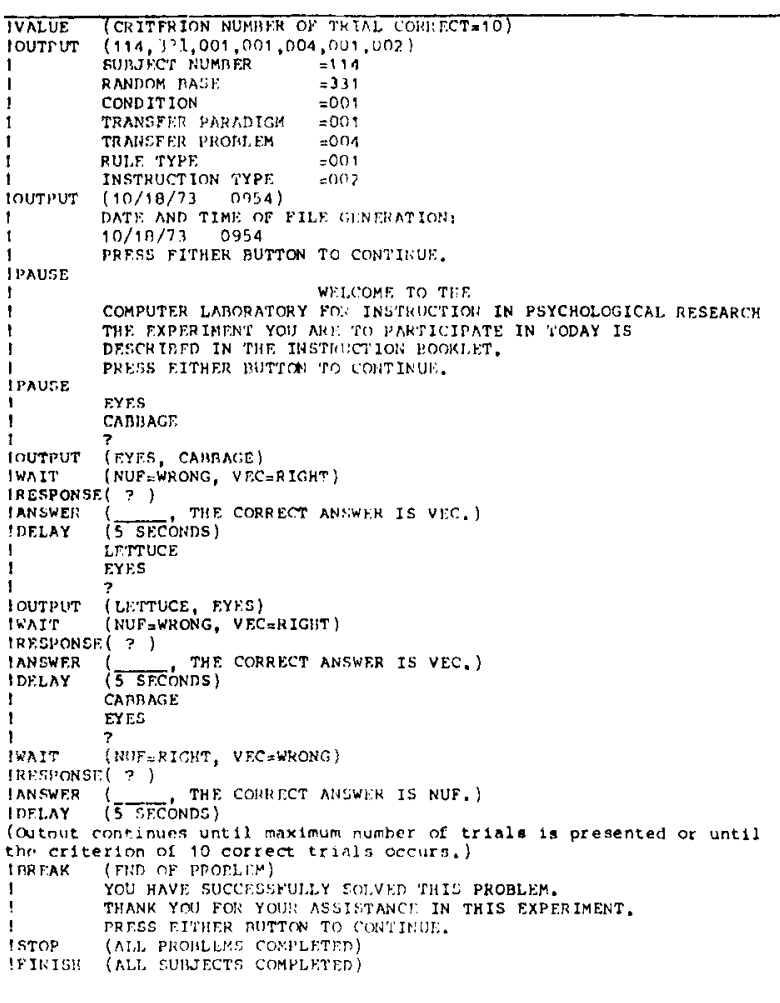

Fig. 2. A sample of output directed to the sequential file by the nonreal-time "generation" program.

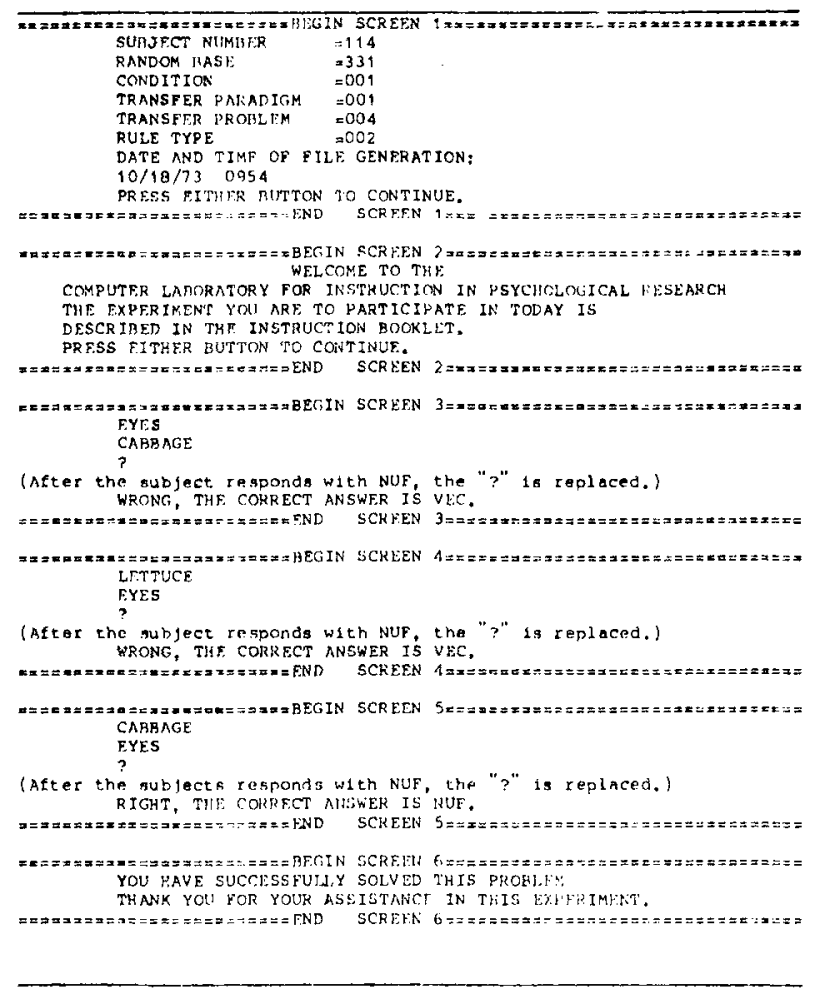

Fig. 3. A sample of output directed to the S's terminal after the real-time "interpreter" program has processed the information on the sequential file presented in Fig. 2.

would be those used to represent the 11 basic real-time operations specified earlier and shown in Fig. 1.

Thus, if the real-time program is to stop presenting stimuli after some number of correct trials, the nonreal-time program would indicate this with the !VALUE command. At each point where output to the S's terminal is to be halted, so that the material can be read, the !PAUSE command would be inserted. Further, if the output is to be delayed or a particular response is sought from the $S$, the !DELAY or !WAIT command would be inserted.

Similarly, when output of information is to be made to the permanent data file or the S's terminal, the !OUTPUT and !(BLANK) commands would be inserted. The !ANSWER command would be inserted at those points where the $S$ is to receive output contingent on his response, and that response would be recorded on the permanent data file by inserting the !RESPONSE command in the sequential file.

Likewise, the real-time program would be informed of the end of the task and all tasks by the !BREAK, !STOP, and !FINISH commands. The !BREAK command is of special interest here, since it signals the end of a single problem. The !STOP command would be inserted at the end of all tasks for a single $\mathbf{S}$. The !FINISH command would be inserted at the end of all tasks for all Ss.

(Note that when the sequential file is generated, the maximum number of possible trials would be output, not just the criterion number. Once the criterion was 


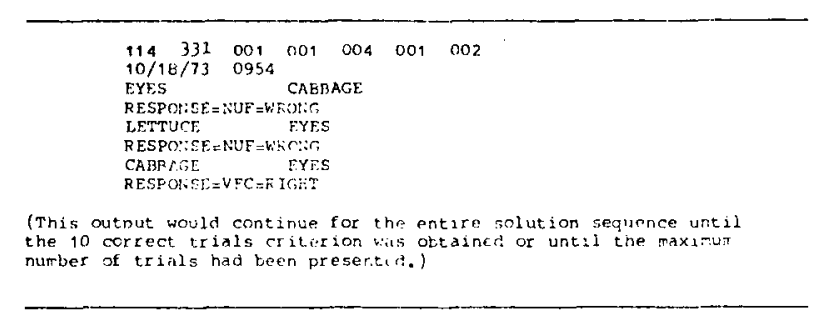

Fig. 4. A sample of output directed to the permanent data file by the real-time "interpreter" program after processing the sequential file information shown in Fig. 2 and after receiving the $S$ responses shown in Fig. 3.

reached, the "extra" information would not be presented, but would be skipped. Therefore, when the criterion is reached, the real-time program would scan through the sequential file, leaving all commands uninterpreted until the first occurrence of the !BREAK command. Commands would once again be interpreted once the !BREAK command was encountered.)

An example of the type of output that would be directed to the sequential file by the nonreal-time "generation" program is shown in Fig. 2.

Once the sequential file was output by the nonreal-time "generation" program, this file would be interpreted in real time by the real-time part of the experiment. The real-time part of the experiment is called the "interpreter" program, since it will interpret each of the 11 commands inserted in the sequential file by the "generation" program. The "interpreter" will input each record from the sequential file and execute the real-time operation specified by the keyword command. In Fig. 3, a sample of the output directed to a S's terminal, as a result of processing the sequential file in Fig. 2, is presented. The responses input by the $\mathrm{S}$ would be requested by the "interpreter" program and processed as specified.

The "interpreter" program would also direct output to the permanent data file as a result of processing the file shown in Fig. 2 and the S's responses indicated in Fig. 3. A sample of several trials of output is presented in Fig. 4.

\section{APPLICATIONS AND CONCLUSIONS}

The multiprogram approach to real-time experimentation has been used to successfully implement a large number of real-time experiments. While many nonreal-time "generation" programs have been developed, only a single real-time "interpreter" program has been developed. Since the "interpreter" program is exceedingly versatile, it has proven to be adequate for most experiments. Because the "interpreter" is small (less than 1,500 locations), several copies of it are simultaneously in operation. The "interpreter" program can be adapted to purposes other than concept identification experiments. Computer-assisted instruction, serial list learning, and virtually any task that stores materials on a sequential file can be implemented. Such implementation only requires a nonreal-time "generation" program to be developed. It is hoped that the reader has acquired some ideas on how to utilize the multiprogram approach to implement new real-time experiments.

Behavior Research Methods \& Instrumentation 1974, Vol. 6, No. 2, 262-266

\title{
Monitoring experimental results*
}

\author{
FRANK RESTLE and W. O. SHAFFER \\ Indiana University, Bloomington, Indiana 47401
}

\begin{abstract}
No matter whether an experiment is a success, a failure, or a mixture of the two, the E needs to see his results immediately to be able to plan his next study. For this purpose, he needs a rapid, simple, relevant analysis of the data. We define a version of this data-summarizing problem and describe a monitor program to solve it. One major decision is what search method and data file organization to use: We use the n-dimensional array method. We specify dependent variables in two parts: first, a measure code like proportion correct, reaction time, etc., and second, a series of coefficients to specify what items will make up the desired dependent measure. Our program is interactive and makes extensive use of default options. Some suggestions are made for the implementation of monitor programs on other hardware.
\end{abstract}

There are three ways an experiment can come out: it can be a failure, a qualified success, or a complete success.

*This project was supported in part by Grant MH 16817 from the United States Public Health Service. The authors appreciate the thoughtful reading of this paper and the comments given by N. John Castellan, Jr.
If the experiment is a complete failure, this may mean, among other things, that all Ss responded at chance, that response times were very long, or that an apparatus failure produced invalid data. A superficial analysis of the overall results will usually reveal such problems, and the $\mathrm{E}$ will want to make some change in 